\title{
High-precision, Temperature Control Based on Grading-structure and PID-feedback Strategies*
}

\author{
Zhiming Xu, ${ }^{1,2)}$ Ming Xu, ${ }^{3) \dagger}$ Wenlong Cheng, ${ }^{1)}$ Hongwu PenG, ${ }^{4)}$ and Yanwei DING ${ }^{2)}$ \\ ${ }^{1)}$ Department of Thermal Science and Energy Engineering, University of Science and Technology of China, Hefei 230027, China \\ ${ }^{2)}$ DFH Satellite Co., Ltd., Beijing 100094, China \\ ${ }^{3)}$ Beihang University, Beijing 100191, China \\ ${ }^{4)}$ Beijing Aerospace Automatic Control Institute, Beijing 100039, China
}

\begin{abstract}
High-precision, temperature control technology is currently an important research field in spacecraft thermal control. High-precision temperature control based on grading-structure and PID-feedback strategies determine by theoretical analysis and grading a thermal control experiment is proposed in this paper. A sensitivity analysis of the key parameters influencing temperature control precision is investigated. The key parameters mainly include the inner emissivity of the transition section $\varepsilon_{t}$, outer emissivity of the central section $\varepsilon_{c}$, effective emissivity from the transition section to the central section $\varepsilon_{i / o}$, inner emissivity of the central section $\varepsilon_{i}$, outer emissivity of the equipment $\varepsilon_{e q}$, outer emissivity of the mounting plate $\varepsilon_{d}$, electronic equipment power $P_{e}$, and the conductivity coefficient of the equipment mounting insulation pad $\lambda$. Both the theoretical and experimental results show that the strategies developed during this research can achieve temperature control precision better than $0.05^{\circ} \mathrm{C}$ ( or $\pm 0.025^{\circ} \mathrm{C}$ ). Parameters $\varepsilon_{t}, \varepsilon_{e q}$, and $\varepsilon_{d}$ not only influence the temperature level, but also influence steady time. $P_{e}, \varepsilon_{c}$, and $\varepsilon_{i}$ only influence the temperature level. $\varepsilon_{i / o}$ not only influences the temperature level, but also influences temperature control precision. Thermal conductivity $\lambda$ influences temperature level rather than temperature control precision because of the active temperature control of the mounting plate. This study provides a new method for the high-precision thermal control of equipment in spacecraft and specifies new directions for future research work.
\end{abstract}

Key Words: Grading Thermal Control, High-precision Temperature Control, Sensitivity Analysis, PID-feedback Strategy

\section{Nomenclature}

$\varepsilon_{t}:$ inner emissivity of the transition section

$\varepsilon_{c}$ : outer emissivity of the central section

$\varepsilon_{i}$ : inner emissivity of the central section

$\varepsilon_{e q}$ : outer emissivity of the equipment

$\varepsilon_{i / o}$ : effective emissivity from transition to central section

$\varepsilon_{d}$ : outer emissivity of the mounting board

$\varepsilon_{c / t}$ : radiant coefficient between transition to central section

$\varepsilon_{e / i}:$ radiant coefficient between equipment to inner of central section

$\varepsilon_{d / t}$ : radiant coefficient between mounting board to transition section

$\varepsilon_{e / d}:$ radiant coefficient between mounting board to equipment

$u(t)$ : active heating power $(\mathrm{W})$

$e(t)$ : temperature difference between set temperature and transient temperature at time

$P_{e}$ : electronic equipment power $(\mathrm{W})$

$P_{c / t}$ : radiant flux from central section to transition section (W)

$P_{\lambda c / t}$ : conductivity flux from central section to transition

(C) 2018 The Japan Society for Aeronautical and Space Sciences

*Received 13 December 2016; final revision received 13 September

2017; accepted for publication 12 December 2017.

†Corresponding author, xuming@buaa.edu.cn section $(\mathrm{W})$

$P_{d / t}$ : radiant flux from the mounting board of central section to transition section (W)

$P_{e / i}$ : inner radiant flux of central section (W)

$P_{i / o}$ : effective radiant flux from central section to transition section $(\mathrm{W})$

$P_{\lambda e / d}$ : conductivity flux from equipment to mounting board (W)

$P_{C m}$ : inner power of electronic equipment $(\mathrm{W})$

$P_{d}$ : radiant flux from electronic equipment to mounting board (W)

$A_{c / t}$ : radiant area between central section to transition section $\left(\mathrm{m}^{2}\right)$

$A_{i / o}$ : radiant area between central section to transition section $\left(\mathrm{m}^{2}\right)$

$A_{e / i}:$ radiant area in central section $\left(\mathrm{m}^{2}\right)$

$A_{d / t}:$ radiant area between central section to transition section $\left(\mathrm{m}^{2}\right)$

$A_{e / d}$ : radiant area between equipment to mounting board $\left(\mathrm{m}^{2}\right)$

$A$ : area of heat insulation pad $\left(\mathrm{m}^{2}\right)$

$T_{t}$ : inner temperature of transition section $\left({ }^{\circ} \mathrm{C}\right)$

$T_{c}$ : outer temperature of central section $\left({ }^{\circ} \mathrm{C}\right)$

$T_{i}$ : inner temperature of central section $\left({ }^{\circ} \mathrm{C}\right)$

$T_{d}$ : mounting board temperature $\left({ }^{\circ} \mathrm{C}\right)$

$T_{e q}$ : temperature of equipment $\left({ }^{\circ} \mathrm{C}\right)$

$K_{P}$ : proportional coefficient 

$T_{D}$ : differential time constant (s)
$T_{I}$ : integration time constant (s)
$T_{s}$ : sampling time (s)
$\lambda$ : conductivity coefficient $\left(\mathrm{W} \cdot \mathrm{m}^{-1} \cdot \mathrm{K}^{-1}\right)$
$\delta$ : thickness of heat insulation pad $(\mathrm{m})$
$\sigma$ : Stefan-Boltzmann constant $\left(\mathrm{W} \cdot \mathrm{m}^{-2} \cdot \mathrm{K}^{-4}\right)$
$C$ : specific heat capacity of equipment $\left(\mathrm{J} \cdot \mathrm{kg}^{-1} \cdot \mathrm{K}^{-1}\right)$
$m$ : mass of equipment $(\mathrm{kg})$

\section{Introduction}

Temperature is the main factor affecting the performance of electronic devices. Proper thermal control solutions are crucial to control the temperatures of electronic devices within acceptable ranges in order to guarantee the long-term stable operation of electronic devices. ${ }^{1)}$ With the development of electronic technologies, requirements for thermal control are also increasing. ${ }^{2}$ Temperature control precision of some devices could reach the millesimal K-level, and indeed call for much higher requirements. In order to precisely measure the gravitational field, the temperature precision of the key device accelerometer of the GRACE satellite launched by NASA in 2002 now reaches $0.1^{\circ} \mathrm{C}$. ${ }^{3)}$ The temperature stability of the key device gravity gradiometer of the GOCE satellite launched by ESA now reaches $10 \mathrm{mK}{ }^{4}{ }^{4}$

At present, high-precision temperature control is achieved applying a variety of methods. Multilayer heat insulation and a large number of independent compensation heaters were used in the thermal control system to achieve a stability requirement of $0.1^{\circ} \mathrm{C}$ per orbit for the GRACE satellite mentioned above. A new method combining structural design with a large number of independent compensation heaters was used in the thermal control system of the GOCE to achieve the stability requirement of $10 \mathrm{mK}{ }^{5}{ }^{5}$ The very stringent high-precision thermal requirements for the BAT detector were achieved by using constantly conductive heat pipes (CCHPs) and loop heat pipes (LHPs). ${ }^{6,7)}$ The precision support structure was constructed of composite materials having a very low coefficient of thermal expansion (CTE), on the order of $10^{-7} / \mathrm{K}$, to meet the very tight thermal stability requirement of the Space Interferometry Mission (SIM). ${ }^{8}$ PID parameters were applied to the self-tuning Active Thermal Control System in order to meet the $\pm 0.05^{\circ} \mathrm{C}$ temperature precision for the optical assembly of the Envisat. ${ }^{9)} \mathrm{A}$ unique passive thermal control enclosure design was proposed for the Gravity Probe-B spacecraft to achieve the requirement of very stringent temperature fluctuation. ${ }^{10)}$ The active thermal control system of the Optical Bench enclosure in the Faint Object Camera can achieve average temperature change of the Optical Bench less than $0.25^{\circ} \mathrm{C} .{ }^{11)}$ A number of actively controlled graphite-epoxy heaters with an isolated thermal design were used to achieve a temperature precision of approximately $0.01^{\circ} \mathrm{C}$ in the Hubble Space Telescope Fine Guidance System and Wave-front Sensor. ${ }^{12)}$ As a heating element, a novel PTC material was introduced to achieve high-precision and adaptive thermal control, and a PTC preparation method with different Curie temperatures was pro- posed. ${ }^{13)}$ Theoretical and experimental studies using a novel PTC material at a room temperature Curie point achieved the highest precision of approximately $0.01{ }^{\circ} \mathrm{C} .{ }^{14)}$ Furthermore, a new thermal control method in which PTC material is combined with a PID control algorithm was proposed to effectively reduce overshoot and increase temperature control precision. ${ }^{15)}$ The thermal design methods described above can achieve high-precision temperature control. However, these design methods increase the complexity, weight and cost of the thermal control system; especially if more precise temperature control is required.

In order to realize high-precision temperature control of spacecraft, a high-precision temperature control method based on grading-structure and PID-feedback strategies is proposed in the paper. The key parameters influencing temperature control precision are derived by theoretical analysis. A sensitivity analysis of the parameters influencing grading thermal control is conducted based on an analytical mathematics model. A small cabin experiment system is built and the grading thermal control method and analytical mathematics model develop are further validated by the experiment results.

\section{High-precision, Temperature Control Based on Grading-structure and PID-feedback Strategies}

\subsection{Grading thermal control and PID-feedback design}

Due to the thermal design requirements of spacecraft, the grading-structure method is usually used. In general, the thermal control method of two different stages is used in the spacecraft thermal design showed in Fig. 1(a). The thermal design method can meet the thermal control requirements of spacecraft without requiring highly precise temperature control. ${ }^{16)}$

In order to investigate high-precision temperature control methods based on grading thermal control, the thermal control method applied to three different stages is taken as an examples in this paper; namely, adding a temperature control

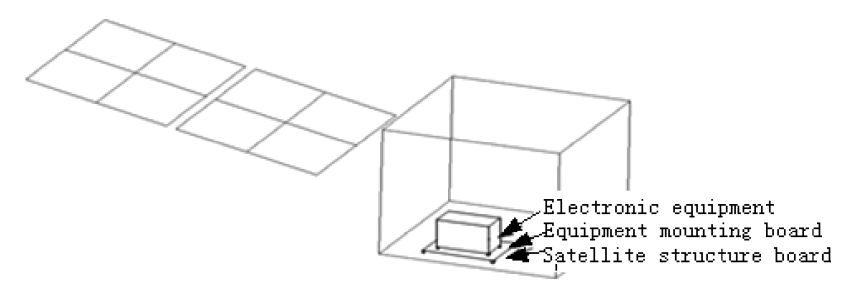

(a) Adopting thermal control method of two different stages

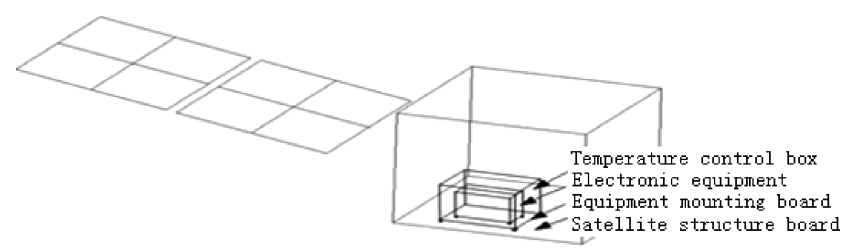

(b) Adopting thermal control method of three different stages

Fig. 1. Comparison of satellite views adopting thermal control methods for two and three different stages. 
box to the external electronic equipment shown in Fig. 1(b). Meanwhile, multi-layer insulation (MLI) material is coated on the exterior of the temperature control box. This design can provide a very stable environment for electronic equipment. Combined with an active high-precision control method on the mounting plate of electronic equipment, it achieves high-precision temperature control. The temperature control method of additional stages can be designed to achieve highly precise temperature control.

The high-precision temperature control method based on the thermal control design for three stages is studied in this paper. The thermal control design utilized for three stages adopts a subpart thermal control design mode according to the satellite's high-precision temperature control requirements. It divides the thermal control system into three categories: central thermal control, transition thermal control, and periphery thermal control. In the three categories, equipment that require the most stable temperature and narrowest work temperature range are placed in the central thermal control section; equipment that requires more stable temperature and a narrower work temperature range are placed in the transition thermal control section; and equipment that doesn't require stable temperature or a narrow work temperature range are placed in the peripheral thermal control section. A compensation heater equipped with a PID algorithm is used in the equipment mounting plate of the central thermal control section. ${ }^{17)}$ Strict heat insulation and independent temperature control are designed for each section in order to reduce the influence of any interaction between them. Figure 2 shows the principle of grading thermal control design.

Figure 3 shows a schematic of the grading thermal control structure that includes the boundary of transition thermal control section, temperature control box (boundary of central thermal control section), electronic equipment, equipment mounting plate, satellite structure plate, and heat insulation pad. As part of the transition thermal control section, the satellite structure board is required to have a good heat dissipation capability. The power of the electronic equipment is mainly dissipated via radiating off the surface of the satellite structure plate. The temperature compensation heaters equipped with the PID algorithm are affixed on the equipment mounting plate.

\subsection{Thermal analysis theory model based on grading thermal control and PID algorithm}

In order to investigate the influence of key parameters on the grading thermal control design method, thermal balance equations can be built according to Figs. 2 and 3. ${ }^{18)}$

A temperature compensation heater equipped with a PID algorithm is affixed to the mounting plate of the electronic equipment. The position of the PID control algorithm adopted is shown in Eq. (1):

$u(t)=K_{P}\left[e(t)+\frac{1}{T_{I}} \sum_{i=0}^{t} e(i) T_{s}+T_{D} \frac{e(t)-e(t-1)}{T_{s}}\right]$

where $K_{P}$ is proportional coefficient, $T_{I}$ is integration time constant, $T_{D}$ is differential time constant, $T_{S}$ is sampling time, $e(t)$ is temperature difference between set temperature and

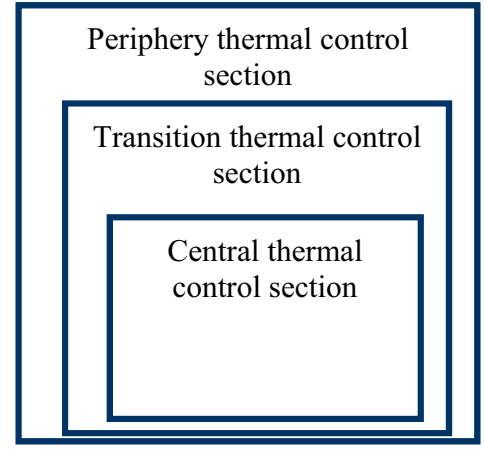

Fig. 2. Schematic of the grading thermal control principle.

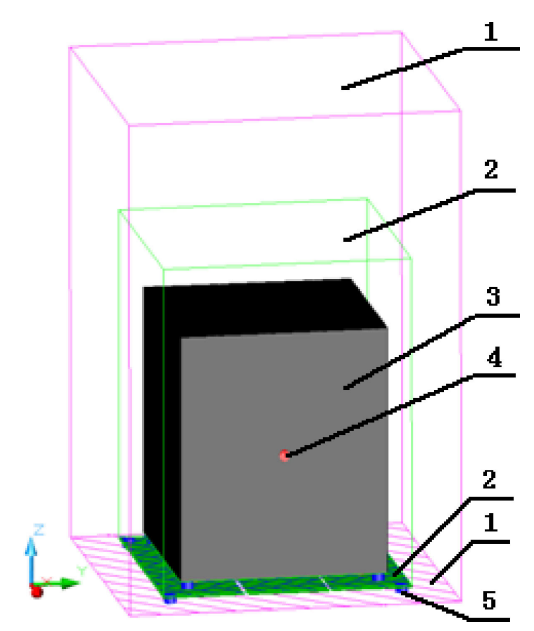

(a) Entire grading thermal control model

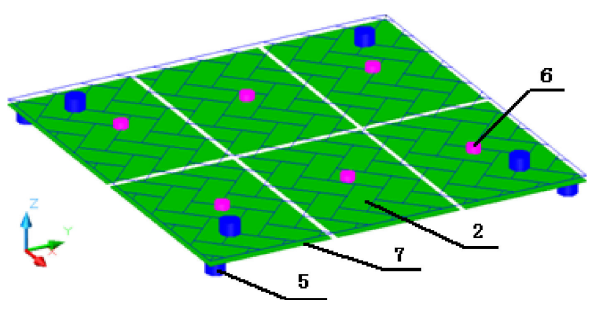

(b) Mounting platform

Fig. 3. Schematic of grading thermal control structure.

(a) Entire grading thermal control model; 1 is transition thermal control section, 2 is temperature control box, 3 is equipment, 4 is temperature measuring point on the equipment, and 5 is the insulation pad. (b) Mounting platform without equipment, 2 and 5 in Fig. 3(a); 6 is the temperature measuring point on the mounting platform and 7 is the heater.

transient temperature at time $t$, and $u(t)$ is active heating power at time $t$.

From the transition thermal control section to the central thermal control section, the heat balance equations are built as shown in Eqs. (2), (3), and (4). Equations (5)-(14) show the radiation and conduction relation:

$$
\begin{aligned}
\int u(t) d t+\int P_{e} d t= & \int P_{c / t} d t+\int P_{\lambda c / t} d t+\int P_{d / t} d t \\
& +\int P_{C m}(t) d t
\end{aligned}
$$

Equation (2) shows the balance equations from the transition thermal control section to the central thermal control 
section. Here, $u(t)$ is active heating power, $P_{e}$ is electronic equipment power, $P_{c / t}$ is radiant flux from the central thermal control section to the transition thermal control section, $P_{\lambda c / t}$ is the conductivity flux from the central thermal control section to the transition thermal control section, and $P_{d / t}$ is the radiant flux from the mounting board of the central thermal control section to the inner surface of the transition thermal control section. $P_{C m}$ is the internal energy of the equipment.

$$
\int P_{c / t} d t=\int P_{i / o} d t+\int P_{e / i} d t
$$

Equation (3) shows the boundary balance equations from the transition thermal control section to the central thermal control section. $P_{c / t}$ is equal to $P_{i / o}$, which is the effective radiant flux from the central thermal control section to the transition thermal control section, and $P_{e / i}$, which is the inner radiant flux of the central thermal control section.

$\int P_{e} d t=\int P_{e / i} d t+\int P_{\lambda e / d} d t+\int P_{d} d t+\int P_{C m}(t) d t$

Equation (4) shows the electronic equipment balance equations for the central thermal control section. $P_{e}$ and $P_{C m}$ are equal to $P_{i / e}$, which is the radiant flux from the inner surface of the central thermal control section to the equipment, $P_{\lambda e / d}$, which is the conductivity flux from equipment to the mounting plate, and $P_{d}$, which is the radiant flux from equipment to the mounting plate.

$$
\begin{aligned}
& P_{c / t}=A_{c / t} \varepsilon_{c / t} \sigma\left(T_{c}^{4}-T_{t}^{4}\right) \\
& P_{i / o}=A_{i / o} \varepsilon_{i / o} \sigma\left(T_{i}^{4}-T_{c}^{4}\right) \\
& P_{e / i}=A_{e / i} \varepsilon_{e / i} \sigma\left(T_{e q}^{4}-T_{i}^{4}\right) \\
& P_{d / t}=A_{d / t} \varepsilon_{d / t} \sigma\left(T_{d}^{4}-T_{t}^{4}\right) \\
& P_{d}=A_{e / d} \varepsilon_{e / d} \sigma\left(T_{e q}^{4}-T_{d}^{4}\right)
\end{aligned}
$$

Equations (5)-(9) show the relation of radiation from the transition thermal control section to the central thermal control section. $A_{c / t}, A_{i / o}, A_{e / i}, A_{d / t}$, and $A_{e / d}$ show the effective radiant area of each section. $\varepsilon_{c / t}, \varepsilon_{i / o}, \varepsilon_{e / i}, \varepsilon_{d / t}$, and $\varepsilon_{e / d}$ show the respective radiant coefficients. $T_{c}, T_{i}, T_{e q}, T_{t}$, and $T_{d}$ show the surface temperature of each section.

$$
\begin{gathered}
P_{\lambda c / t}=A \lambda\left(T_{d}-T_{t}\right) / \delta \\
P_{\lambda e / d}=A \lambda\left(T_{e q}-T_{d}\right) / \delta \\
P_{C m}(t)=C m \frac{d T_{e q}}{d t}
\end{gathered}
$$

Equations (10) and (11) show the conductivity relation from the transition thermal control section to the central thermal control section. $A$ is the effective conductivity area and $\lambda$ is the conductivity coefficient. Equation (12) shows the change in equipment inner power.

$$
\begin{aligned}
& \varepsilon_{e / i}=\frac{1}{\frac{1}{\varepsilon_{i}}+\frac{1}{\varepsilon_{e q}}-1} \\
& \varepsilon_{c / t}=\frac{1}{\frac{1}{\varepsilon_{c}}+\frac{1}{\varepsilon_{t}}-1} \\
& \varepsilon_{e / d}=\frac{1}{\frac{1}{\varepsilon_{e q}}+\frac{1}{\varepsilon_{d}}-1}
\end{aligned}
$$

Equations (13)-(15) show the effective emissivity relation from the transition thermal control section to the central thermal control section. $\varepsilon_{c}, \varepsilon_{t}, \varepsilon_{i}, \varepsilon_{c / t}, \varepsilon_{e q}$, and $\varepsilon_{d}$ are the central area outer emissivity, transition area inner emissivity, central area inner emissivity, effective emissivity from the transition thermal control section to the central thermal control section, and electronic equipment outer emissivity and mounting plate outer emissivity from the transition thermal control section to the central thermal control section, respectively.

The above theory analyses show that the main parameters that influence temperature control precision are $\varepsilon_{c}, \varepsilon_{t}, \varepsilon_{i}, \varepsilon_{c / t}$, $\varepsilon_{e q}, \varepsilon_{d}, m, \lambda, P_{e}$, and $u(t)\left(K_{P} / T_{I}(s) / T_{D}(s)\right)$.

\subsection{Sensitivity analyses of key parameters influencing grading thermal control}

A simulation mathematics model was built according to Fig. 3. The influence of $\varepsilon_{c}, \varepsilon_{t}, \varepsilon_{i}, \varepsilon_{i / o}, \varepsilon_{e q}, \varepsilon_{d}, m, \lambda, P_{e}$, and $u(t)\left(K_{P} / T_{I}(s) / T_{D}(s)\right)$ on grading thermal control are discussed.

According to the grading thermal design principle and the experience data of satellites in orbit, ${ }^{16)}$ the temperature range of $-15^{\circ}-+50^{\circ} \mathrm{C}$ and fluctuation allowance of $\pm 2.5^{\circ} \mathrm{C}$ from the periphery thermal control section to the transition thermal control section can be achieved using the general thermal control design method. In order to investigate a high-precision temperature control method based on grading thermal control from the transition thermal control section to the central thermal control section, the following assumption is proposed: the boundary temperature changes of the transition thermal control section are simulated through six faces of the transition thermal control section boundary in Fig. 3, as shown in Table 1.

Table 2 shows the range of change and influence analysis results of $\varepsilon_{c}, \varepsilon_{t}, \varepsilon_{i}, \varepsilon_{i / o}, \varepsilon_{e q}, \varepsilon_{d}, m, \lambda, P_{e}$, and $u(t)\left(K_{P} /\right.$ $\left.T_{I}(s) / T_{D}(s)\right)$. Analysis results are shown in Figs. 4-8.

Figure 4 shows the analysis results of $\varepsilon_{i / o}$ with change ranging from 0.03 to 0.9 . The analysis results show that $\varepsilon_{i / o}$

Table 1. Boundary temperature of the transition thermal control section.

\begin{tabular}{lc}
\hline Position & Control temperature \\
\hline Negative $X$ & $-10 \pm 2.5^{\circ} \mathrm{C}$ \\
Positive $X$ & $15 \pm 2.5^{\circ} \mathrm{C}$ \\
Negative $Y$ & $35 \pm 2.5^{\circ} \mathrm{C}$ \\
Positive $Y$ & $15 \pm 2.5^{\circ} \mathrm{C}$ \\
Negative $Z$ & $5 \pm 2.5^{\circ} \mathrm{C}$ \\
Positive $Z$ & $15 \pm 2.5^{\circ} \mathrm{C}$ \\
\hline
\end{tabular}


Table 2. Parameter change range and influence analysis results. (“-” represents no influence, “ $\checkmark$ "represents influence)

\begin{tabular}{lcccc}
\hline & $\begin{array}{c}\text { Parameter } \\
\text { value } \\
\text { Parameters }\end{array}$ & \multicolumn{4}{c}{ Influence } \\
\cline { 3 - 5 } & change & $\begin{array}{c}\text { Temperature } \\
\text { control } \\
\text { range }\end{array}$ & $\begin{array}{c}\text { Temperature } \\
\text { precision }\end{array}$ & $\begin{array}{c}\text { Balance } \\
\text { time }\end{array}$ \\
\hline$\varepsilon_{i / o}$ & $0 \rightarrow 1$ & $\checkmark$ & $\checkmark$ & $\checkmark$ \\
$\varepsilon_{c}$ & $0 \rightarrow 1$ & - & $\checkmark$ & - \\
$\varepsilon_{t}$ & $0 \rightarrow 1$ & - & $\checkmark$ & $\checkmark$ \\
$\varepsilon_{i}$ & $0 \rightarrow 1$ & - & $\checkmark$ & \\
$\varepsilon_{e q}$ & $0 \rightarrow 1$ & - & $\checkmark$ & $\checkmark$ \\
$\varepsilon_{d}$ & $0 \rightarrow 1$ & - & $\checkmark$ & $\checkmark$ \\
$\lambda$ & $0.5 \rightarrow 121$ & $\checkmark$ & $\checkmark$ & - \\
$P_{e}$ & $0 \rightarrow 10$ & - & $\checkmark$ & - \\
$m$ & $3 \rightarrow 8.5$ & $\checkmark$ & - & $\checkmark$ \\
$u(t)\left(K_{P} /\right.$ & $3(1 / 6 / 3) \rightarrow$ & $\checkmark$ & - & - \\
$\left.T_{I}(s) / T_{D}(s)\right)$ & $8(10 / 3 / 3)$ & & & \\
\hline
\end{tabular}

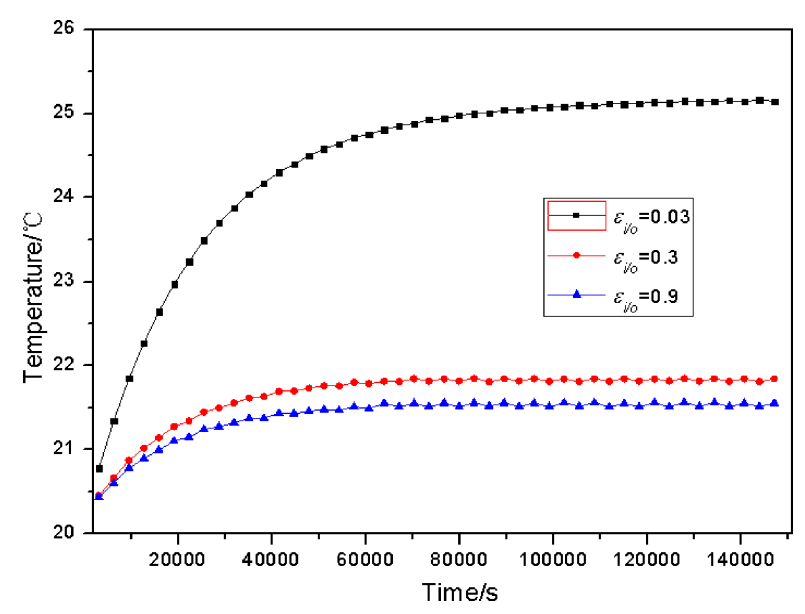

Fig. 4. Influence analysis results of the parameters $\varepsilon_{i / o}$.

not only influences temperature level, but also influences temperature control precision and balance time. Temperature control precision becomes worse as $\varepsilon_{i / o}$ increases.

Figure 5 shows the analysis results of changing $\varepsilon_{c}, \varepsilon_{t}, \varepsilon_{i}$, $\varepsilon_{e q}, \varepsilon_{d}$, and $\lambda$, compared with the analysis results of no parameter change. The analysis results show $\varepsilon_{t}, \varepsilon_{e q}$, and $\varepsilon_{d}$ not only influence the level of temperature, but also steady time. $\varepsilon_{c}$ and $\varepsilon_{i}$ only influence the level of temperature. The conductivity coefficient $\lambda$ influences temperature level more than temperature control precision because of the active temperature control in the mounting plate.

Figures 6, 7, and 8 show influence analysis results of changing $P_{e}, m$, and $u(t)\left(K_{P} / T_{I}(s) / T_{D}(s)\right)$. Figure 6 shows that electronic equipment power influences the temperature level from $+22.45^{\circ} \mathrm{C}$ to $+32.86^{\circ} \mathrm{C}$ as electronic equipment power increases from $4 \mathrm{~W}$ to $9 \mathrm{~W}$. Figure 7 shows that heat capacity changes as the mass of the electronic equipment changes, and influences temperature control precision and balance time of the electronic equipment. Figure 8 shows that compensation heaters with PID algorithms influence the temperature control precision and can achieve higher temperature control precision with the appropriate choice of PID parameters. The size of the active heating power $u(t)$ only influences the duty cycle of the heating time.

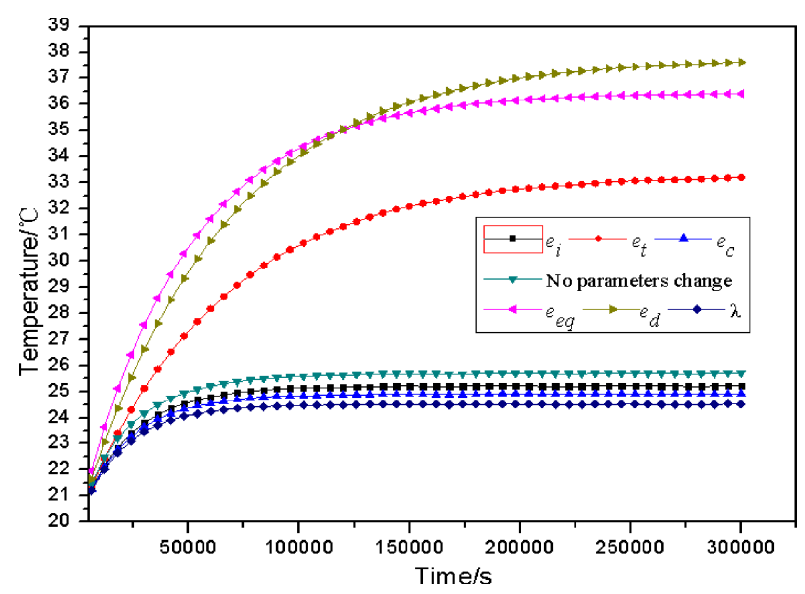

Fig. 5. Influence analysis results of $\varepsilon_{c}, \varepsilon_{t}, \varepsilon_{i}, \varepsilon_{e q}, \varepsilon_{d}$, and $\lambda$.

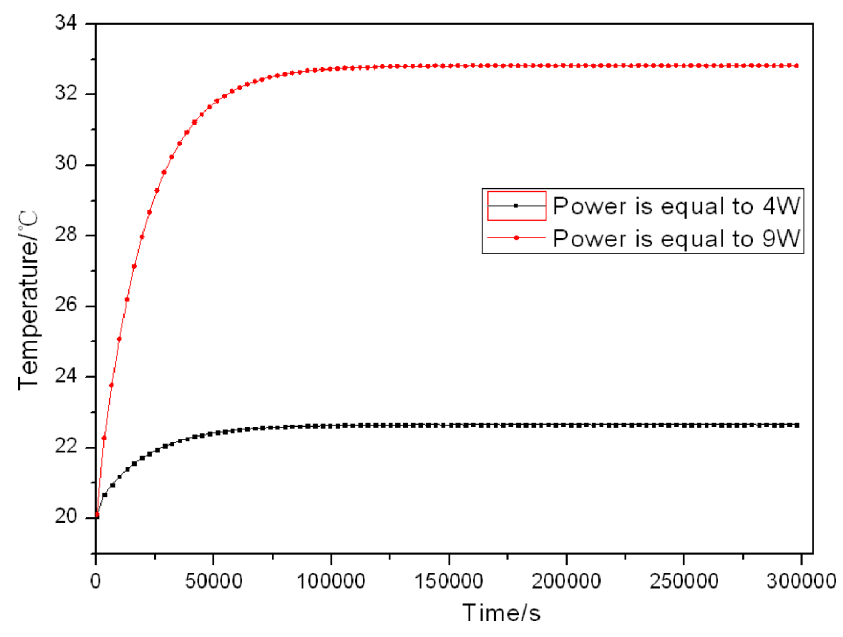

Fig. 6. Influence analysis results of the parameters $P_{e}$.

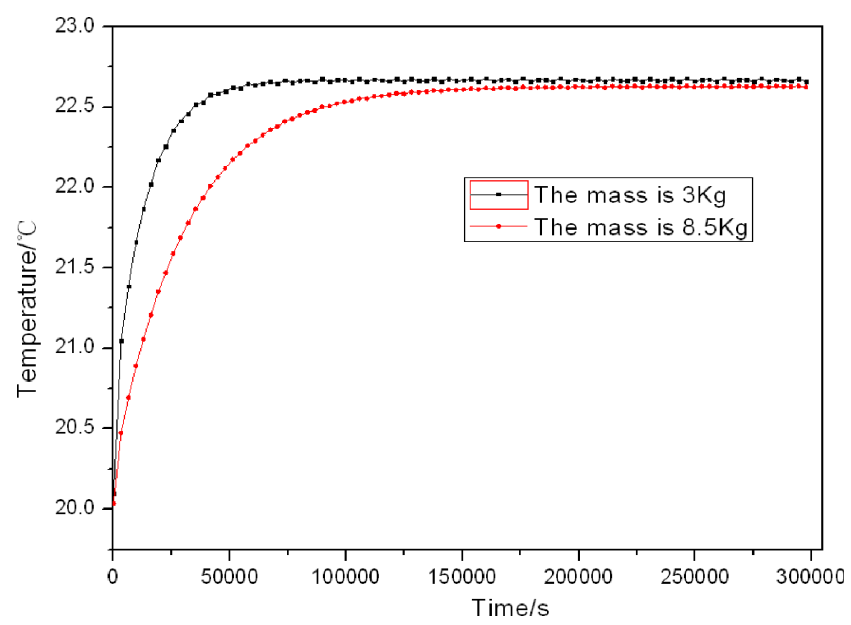

Fig. 7. Influence analysis results of the parameters $m$.

Figure 9 shows that a temperature precision of better than $\pm 0.01^{\circ} \mathrm{C}$ can be achieved by grading thermal control, while temperature precision of $\pm 0.15^{\circ} \mathrm{C}$ is achievable without grading thermal control. Temperature fluctuation of the environment outside and inside the temperature control box was reduced from $\pm 1.85^{\circ} \mathrm{C}$ to $\pm 0.025^{\circ} \mathrm{C}$ when using grading 


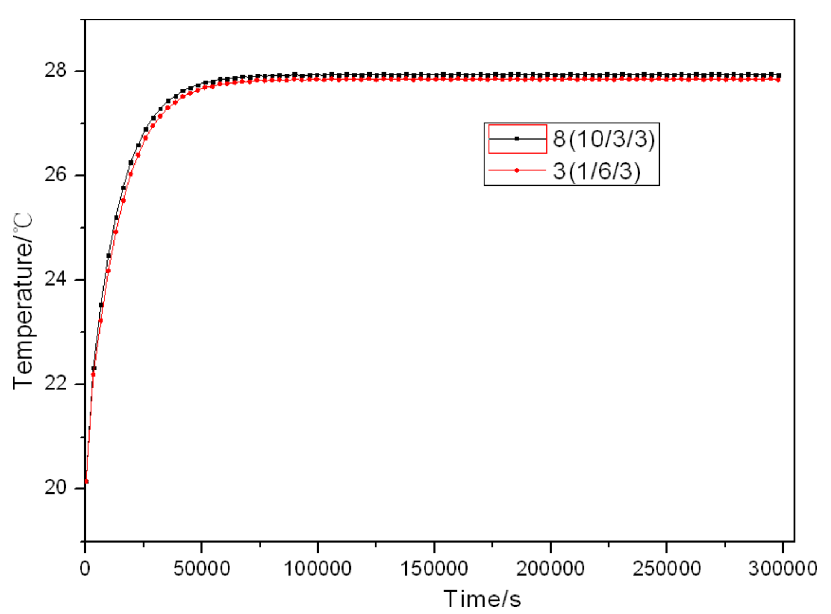

Fig. 8. Influence analysis results of different $u(t)\left(K_{P} / T_{I}(s) / T_{D}(s)\right)$.

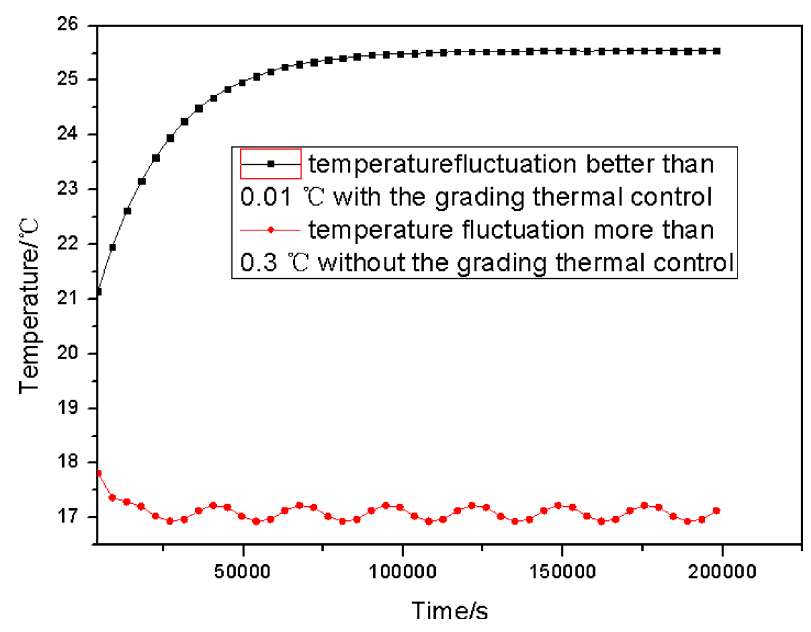

Fig. 9. Influence analysis results based on grading thermal control.

thermal control, as shown in Fig. 10. The temperature control box design can provide a very stable environment for the equipment.

The above analysis results show that a material with smaller effective emissivity $\varepsilon_{i / o}$ can be selected to improve temperature precision. Therefore, smaller emissivity $\varepsilon_{c}$ can be chosen to control the temperature level. A larger emissivity $\varepsilon_{t}, \varepsilon_{e q}$, and $\varepsilon_{d}$ can be chosen to control the temperature level and balance time. Larger emissivity coefficient $\varepsilon_{t}$ can be chosen to control the temperature level. A larger $P_{e}$ can be chosen to attain a higher temperature level. A larger $m$ can be chosen to control temperature control precision and balance time. A larger $\lambda$ can be chosen to control temperature control precision and temperature level. Therefore, the simulation and analysis results indicate that the grading thermal control method can provide a very stable environment for equipment and can achieve highly precise control.

\section{Experiment Based on Grading Thermal Control and PID Algorithm}

\subsection{Experimental system}

An experimental system was built based on the grading thermal control structure schematic shown in Fig. 3 and

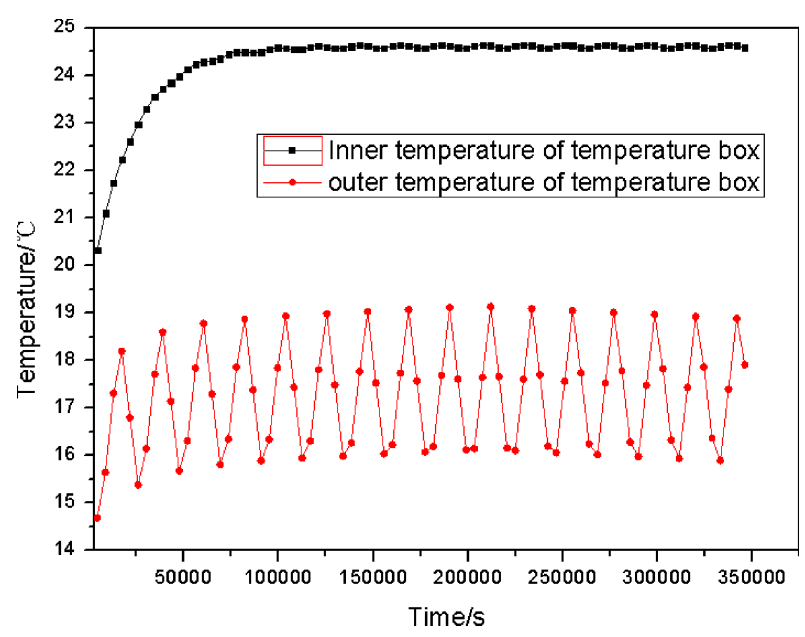

Fig. 10. Simulation analysis results of environment fluctuation.

the thermal analysis mathematics model in Section 2.3. The experimental system consisted of a vacuum simulator, boundary simulated cabin of the transition thermal control section, temperature control box, electronic equipment, temperature control system and experimental acquisition system.

The experiment was conducted in a large vacuum simulator. A vacuum simulator of $2.0 \mathrm{~m}$ (diameter) $\times 2.5 \mathrm{~m}$ (length) was used. The vacuum in the simulator was better than $1.3 \times 10^{-3}$, and temperature of the heat sink was lower than $-80^{\circ} \mathrm{C}$. The experimental acquisition system gathered data from pt100 s. The sampling time is $1 \mathrm{~s}$.

According to Fig. 3, an aluminum plate $22 \times 22 \times$ $0.5 \mathrm{~cm}$ in size was used as the electronic equipment mounting plate, base plate of the temperature box. Six pt100s were uniformly affixed on one side of the electronic equipment mounting plate. Six active temperature control compensation heaters equipped with a high-precision PID algorithm were uniformly affixed on the other side of the mounting plate. One pt100 was affixed to electronic equipment. The other five sides of the temperature box, with the size of $22 \times$ $22 \times 23 \mathrm{~cm}$ were installed on the electronic equipment mounting plate. The temperature control box was laid in a simulation cabin, with the size of $42 \times 35 \times 35 \mathrm{~cm}$. Heaters were affixed to six surfaces of the cabin to simulate boundary temperature changes of the transition thermal control section.

\subsection{Thermal control design of grading thermal control structure system}

The thermal control design of the grading thermal control structure shown in Fig. 3 was determined based on sensitivity analysis results of key parameters that influenced grading thermal control in Section 2.3. According to the parameters range of change and influence analysis results shown in Table 2, and based on thermal control materials that can be easily attained, the grading thermal control structure thermal control methods are shown in Table 3. The thermal control design is explained below.

(1) The outside of the thermal control box was covered with a multilayer heat insulation subassembly and alumi- 
Table 3. Parameters and corresponding material choice.

\begin{tabular}{lll}
\hline \multicolumn{1}{c}{ Parameters } & \multicolumn{1}{c}{ Parameter value } & \multicolumn{1}{c}{ Property } \\
\hline$\varepsilon_{i / o}$ & 0.03 & Multilayer subassembly \\
$\varepsilon_{c}$ & 0.1 & Aluminized polyester film \\
$\varepsilon_{t}$ & 0.85 & Black lacquer \\
$\varepsilon_{i}$ & 0.85 & Black lacquer \\
$\varepsilon_{e q}$ & 0.85 & Black lacquer \\
$\varepsilon_{d}$ & 0.85 & Black lacquer \\
$\lambda$ & $0.5 \mathrm{~W} \cdot \mathrm{m}^{-1} \cdot \mathrm{K}^{-1}$ & Fiberglass insulation pads \\
$P_{e}$ & $6.7 \mathrm{~W}$ & Design \\
$m$ & $5.5 \mathrm{~kg}$ & Design \\
$u(t)\left(K_{P} / T_{I}(s) / T_{D}(s)\right)$ & $5 \mathrm{~W}(1 / 7 / 3)$ & Design \\
\hline
\end{tabular}

nized polyester film. The inside of temperature control box was lacquered black, as shown in Fig. 11(a).

(2) All surfaces of the simulation cabin were coated with black lacquer. The heaters were affixed on the outer surfaces of the simulation cabin to simulate the change in heat flux of the transition thermal control section based on Table 1 and shown in Fig. 11(b).

(3) The electronic equipment was lacquered black. The power of the electronic equipment was $6.7 \mathrm{~W}$.

(4) Fiberglass insulation pads were used between the electronic equipment and the mounting board, as well as between the mounting board and structural board.

(5) High-precision temperature compensation heaters equipped with PID algorithms were affixed to the mounting plate of the electronic equipment. The PID control algorithm adopted the position PID control algorithm shown in Eq. (1).

(6) The experimental acquisition system is shown in Fig. 11(c). The sampling time was $1 \mathrm{~s}$.

\section{Theoretical and Experimental Results}

Figure 12(a) shows that the experimental temperature control precision of the equipment mounting plate is $\pm 0.05^{\circ} \mathrm{C}$. Figure 12 (b) shows the simulation temperature control precision results, proving the equipment mounting plate can achieve $\pm 0.03^{\circ} \mathrm{C}\left(18.56 \pm 0.03^{\circ} \mathrm{C}\right)$. Figure 13 (a) shows that the experimental temperature control precision of the equipment is $\pm 0.025^{\circ} \mathrm{C}$. Figure 13 (b) shows the simulation temperature control precision results, proving the equipment can achieve better than $\pm 0.01^{\circ} \mathrm{C}$ (i.e., $25.547 \pm$ $0.01^{\circ} \mathrm{C}$ ). A comparison of results is shown in Table 4 .

Compared to the experimental results, the simulation analysis results were for simulation model simplification. The experimental errors were due to setting up the thermal implementation process itself and uncertainties in material properties. Therefore, the experiment results were reasonable and the target of highly precise temperature control was achieved. The results show that the experimental data is in reasonable agreement with the simulation data shown in Table 4 . The simulation model was verified using experiment results. Therefore, a thermal design based on grading-structure and PID-feedback strategies is an effective method for solving high-precision temperature control.

In this research, temperature control precision of $0.05^{\circ} \mathrm{C}$ was achieved by building a temperature control box around

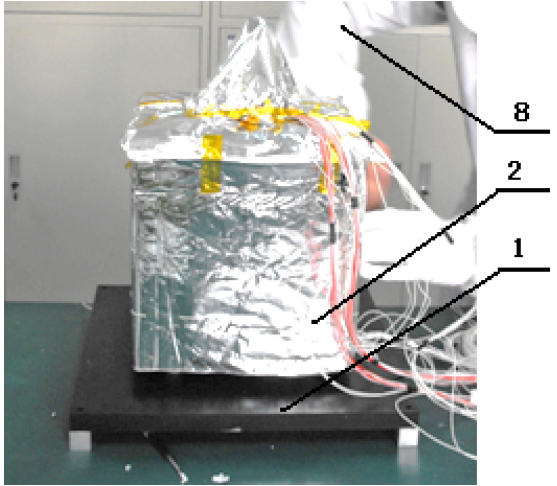

(a) Temperature control box

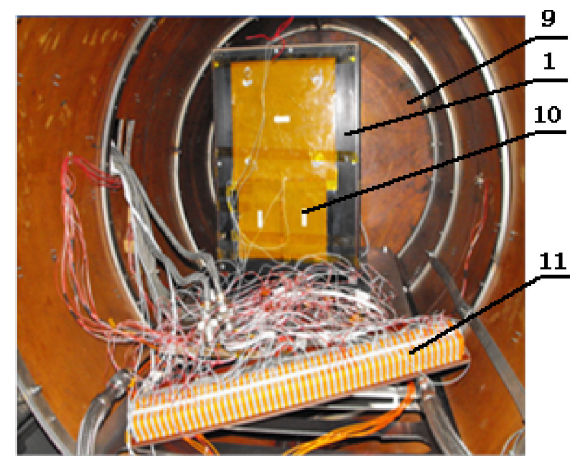

(b) Vacuum simulator

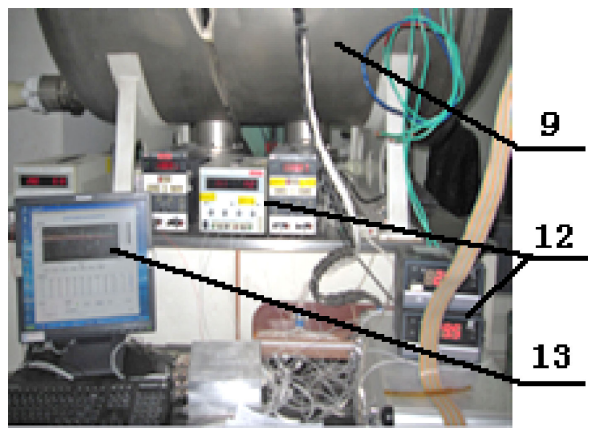

(c) Data acquisition system

Fig. 11. The experimental system.

(a) Temperature control box; 1 is the satellite structure board as part of the transition section, 2 is the temperature control box with a silver-white multilayer heat insulation subassembly, and 8 is the operator. (b) Vacuum simulator; 1 is the transition section substituted by the simulation cabin coated with black lacquer, 9 is the vacuum simulator, 10 is the heaters, and 11 is the data line flashboard. (c) Data acquisition system; 9 is the vacuum simulator, 12 is the data acquisition system, and 13 is the data computer.

the equipment using PID-feedback strategies for the mounting plate. The entire temperature control box, including electronic equipment, is set on the radiating structural surface of a satellite using an insulation pad; the outside of which is covered by a multilayer heat insulation subassembly. Visibly, thermal control implementation is simple and the thermal control material can be easily obtained. Therefore, compared to other design methods, the method developed and proposed in this paper results in a reduction in complexity, weight and cost of the thermal control system, which further improves the reliability of the thermal control system. 


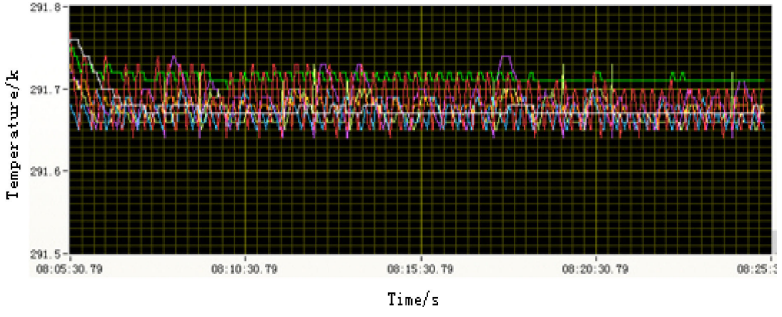

(a) Experiment curve for the mounting plate

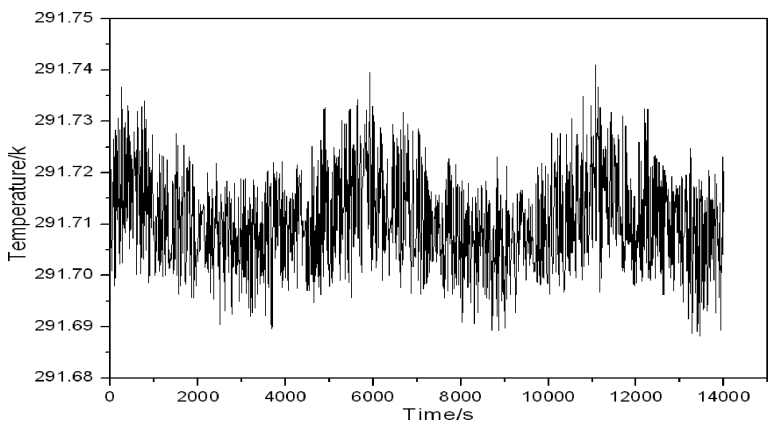

(b) Simulation curve for the mounting plate

Fig. 12. Temperature curve for the equipment mounting plate.

Table 4. The temperature balance for simulation and experiment $\left({ }^{\circ} \mathrm{C}\right)$.

\begin{tabular}{lcc}
\hline Temperature & Electronic equipment & Mounting board \\
\hline Simulation & $25.547 \pm 0.01$ & $18.56 \pm 0.03$ \\
Experiment & $25.97 \pm 0.025$ & $18.56 \pm 0.05$ \\
\hline
\end{tabular}

\section{Conclusions}

The present work studied a high-precision thermal control method based on grading-structure and PID-feedback strategies. The thermal control methods for three different stages were taken as an example and investigated. An analytical model for grading thermal control was built and analyzed using a system simulation analysis method. Parameters influencing temperature control precision were analyzed using theoretical analysis. The sensitivity analyses of the parameters influencing grading thermal control were investigated based on the analytical model. Finally, a small cabin experiment system was built and the grading thermal control method and analytic mathematics model were further validated. The results of this study are concluded as follows:

1) The thermal design based on grading-structure and PID-feedback strategies is an effective method to achieve spacecraft high-precision temperature control.

2) The method developed and reported in the paper can achieve temperature control precision better than $0.05^{\circ} \mathrm{C}$ (or $\pm 0.025^{\circ} \mathrm{C}$ ).

3) Parameters $\varepsilon_{t}, \varepsilon_{e q}$, and $\varepsilon_{d}$ not only influence the level of temperature, but also steady time; parameters $P_{e}, \varepsilon_{c}$, and $\varepsilon_{i}$ only influence the level of temperature; parameter $\varepsilon_{i / o}$ not only influences the level of temperature, but also temperature control precision; the conductivity coefficient $\lambda$ influences the level of temperature more than the precision of temperature control because of the mounting plate active

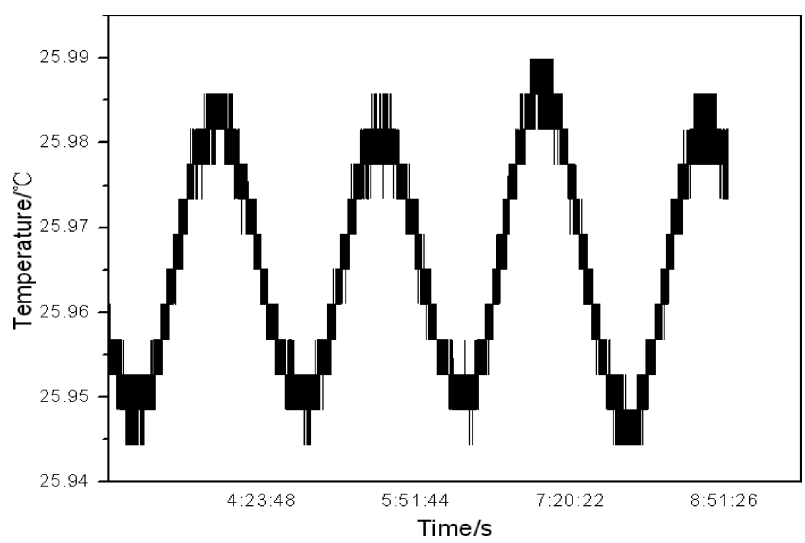

(a) Experimental curve for the equipment

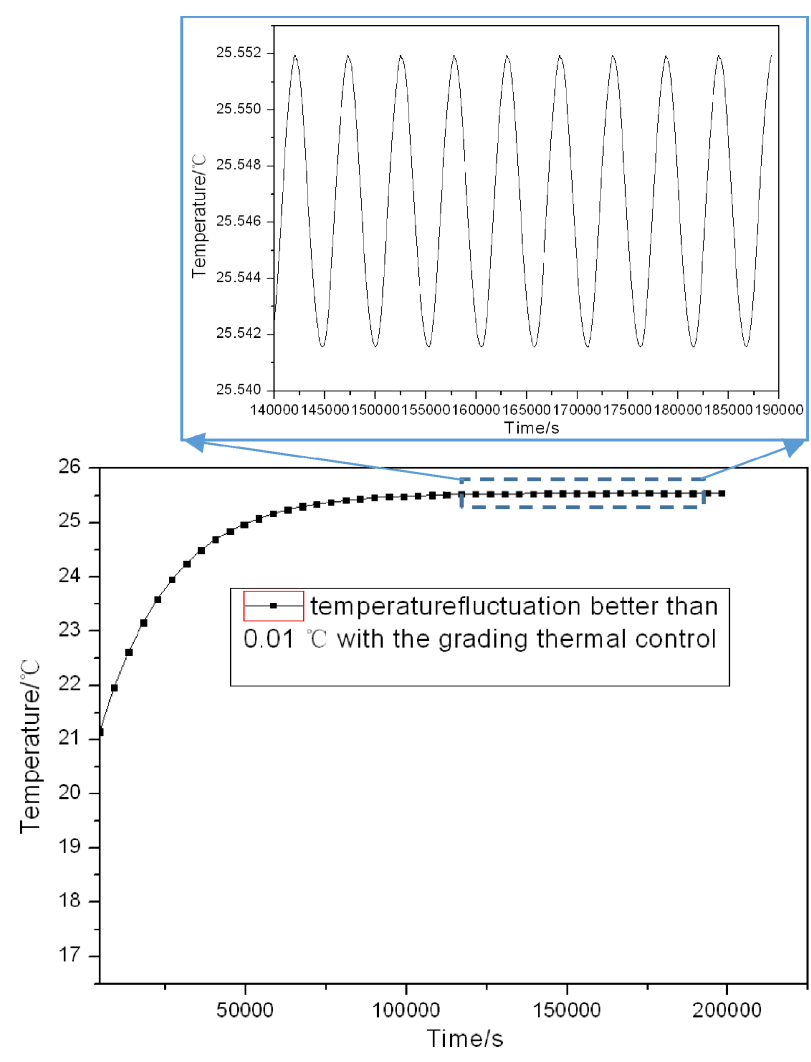

(b) Simulation curve for the equipment

Fig. 13. Temperature curve for the equipment.

temperature control.

4) The sensitivity analysis results supply a reference on materials choice for spacecraft thermal design using the grading thermal control method.

\section{Acknowledgments}

This work is supported by the National Science Foundation of China (41274041 and 11772024), Beijing Natural Science Foundation (4153060), the Knowledge Innovation Project of the Chinese Academy of Sciences (grant no. KJCX1-YW-021), and the Fundamental Research Funds for the Central Universities and China Scholarship Council program. 


\section{References}

1) Cheng, W. L., Liu, N., and Wu, W. F.: Studies on Thermal Properties and Thermal Control Effectiveness of a New Shape-stabilized Phase Change Material with High Thermal Conductivity, Appl. Therm. Eng., 36 (2012), pp. 1-10.

2) Ryan, A. S.: Overview of NASA's Thermal Control System Development for Exploration Project, 40th International Conference on Environmental Systems, AIAA 2010-6135, 2010.

3) Adam, D.: Gravity Measurement Amazing GRACE, Nature, 416 (2002), pp. 523-525.

4) Ji, B., Bian, S. F., and Hu, Q. F.: Introduction of GOCE Satellite and Its Satellite Gravity Gradiometer, Space Int., 11 (2004), pp. 30-32.

5) GOCE (Gravity Field \& Steady-State Ocean Circulation) System Critical Review (CDR), May 2005.

6) Choi, M. K.: Thermal Design to Meet Stringent Temperature Gradient/Stability Requirements of SWIFT BAT Detectors, AIAA, Washington, 2000, pp. 2000-2096.

7) Choi, M. K.: Thermal Assessment of SWIFT Instrument Module Thermal Control System and Mini Heater Controllers after 5+ Years in Flight, 40th International Conference on Environmental Systems, AIAA 2010-6003, 2010.

8) Aaron, K. M., Hashemi, A., Morris, P., and Nienberg, J.: Space Interferometry Mission (SIM) Thermal Design, Interferometry in Space 2003-4852, pp. 49-58.

9) Lemmen, M., Brocker, M., and Polzer, J.: In-Flight Results of the Sciamachy Optical Assembly Active Thermal Control System, 34th International Conference on Environmental Systems (ICES), Colorado Springs, Colorado, July, 2004.

10) Hsu, I., Shokralla, S., and Calhoon, S.: Thermal Control Enclosure for the Forward Electronics of Gravity Probe-B Spacecraft, 30th AIAA
Thermophysics Conference, San Diego, CA, AIAA 95-2017, 1995.

11) Jaekel, E. K. J., Erne, W., and Soulat, G.: The Thermal Control System of the Faint Object Camera (FOC), AIAA 15th Thermophysics Conference, Snowmass, Colorado, AIAA 80-1501, 1980.

12) Stoll, R. and Williams, L.: Thermal Design and Testing of the Hubble Space Telescope Fine Guidance System and Wavefront Sensor, AIAA/ASME 4th Joint Thermophysics and Heat Transfer Conference, Boston, Massachusetts, June 1986.

13) Cheng, W. L., Yuan, S., and Song, J. L.: Studies on Preparation and Adaptive Thermal Control Performance of Novel PTC Material with Controllable Curie Temperatures, Energy, 74 (2014), pp. 447-454.

14) Cheng, W. L., Song, J. L., Liu, Y., Yuan, S., Wu, W. F., and Xu, Z. M.: Theoretical and Experimental Studies on Thermal Control by Using a Novel PTC Material with Room Temperature Curie Point, Int. J. Heat Mass Transfer, 74 (2014), pp. 441-447.

15) Song, J. L., Cheng, W. L., Xu, Z. M., Yuan, S., and Liu, M. H.: Study on PID Temperature Control Performance of a Novel PTC Material with Room Temperature Curie Point, Int. J. Heat Mass Transfer, 64 (2015), pp. 343-351.

16) Gilmore, D. G.: Satellite Thermal Control Handbook, The Aerospace Corporation Press, El Segundo, California, 2002, pp. 639-666.

17) Deng, W., Zhang, Y., and Chen, J. H.: Optimization and Realization of Digital Temperature Control System for CPT Atomic Frequency Standard, Acta Electron. Sinica, 40 (2012), pp. 1889-1892.

18) Hou, Z. Q. and Hu, J. G.: Spacecraft Thermal Control Technology-Theory and Application, Chinese Science and Technology Press, Beijing, 2007.

Y. Miyazaki Associate Editor 